Supplement of Biogeosciences, 15, 5061-5091, 2018

https://doi.org/10.5194/bg-15-5061-2018-supplement

C Author(s) 2018. This work is distributed under

the Creative Commons Attribution 4.0 License.

(c) (1)

Supplement of

\title{
Mesoscale contribution to the long-range offshore transport of organic carbon from the Canary Upwelling System to the open North Atlantic
}

Elisa Lovecchio et al.

Correspondence to: Elisa Lovecchio (elisa.lovecchio@usys.ethz.ch)

The copyright of individual parts of the supplement might differ from the CC BY 4.0 License. 


\section{S 1 Upwelling filaments identification algorithm}

\section{S 1.1 Algorithm description}

Our newly developed filament detection algorithm targets the identification of so-called "upwelling filaments", i.e., structures that originate in the nearshore of an upwelling system and extend towards the open sea in the form of small-scale narrow streams that export upwelled cold water offshore. The algorithm uses only sea surface temperature (SST) as the basis for the detection, i.e., it does not include surface chlorophyll (S-CHL), which is commonly used for the identification of these structures from satellite images (Gabric et al., 1993; Cravo et al., 2010). Limiting the algorithm to use SST alone results in a more general algorithm that works when S-CHL is not available (e.g., due to cloud cover or because of the model not including a biological submodel). Further, it allows us to avoid the possible self-referencing loop of already defining filaments as organic carbon rich structures. However, in the successive evaluation of the algorithm performance we also used S-CHL images, as chlorophyll constitutes a good tracer for the identification of the pathways of upwelled water.

We describe here the successive steps that constitute our new filament identification routine. The algorithm itself is available from the author upon request.

The routine requires as input an SST $(\mathrm{x}, \mathrm{t})$ field, with $\mathrm{x}$ representing the discrete space $\left(\mathrm{x}_{i, j}\right)$ covered by the grid, and t time. The algorithm expects the that the temporal resolution of the SST field is of the order daily to weekly. This SST time series must extend over a sufficiently long time, so that a climatological reference mean $\overline{\operatorname{SST}}(\mathrm{x}, \mathrm{t})$ field for each time step can be generated, if not already available. In our study we used as a reference mean $\overline{\mathrm{SST}}(\mathrm{x}, \mathrm{t})$ the long-term climatological monthly mean SST field calculated from the full period of analysis (24 years) and linearly interpolated in time to 2-day steps, the same time resolution of our analyzed SST(x,t).

The algorithm requires 2 user-defined parameters. These are ND, i.e., the degrees of latitude/longitude defining the level of spatial smoothing used to smooth the reference mean $\overline{\operatorname{SST}}(\mathrm{x}, \mathrm{t})$ field, and $\Delta \mathrm{SST}^{\mathrm{TH}}$, i.e., the SST threshold to separate the filament from the background. These parameters need to tuned depending on the regional properties of the offshore SST gradient and on the resolution of the analyzed SST field.

The algorithm proceeds in 6 steps. In the first step, the climatological reference is computed from SST(x,t) for each time-step of the analysis, resulting in the $\overline{\operatorname{SST}}(\mathrm{x}, \mathrm{t})$ field. In step 2, this field is smoothed using a bin averaging filter (Figure 1). This is achieved by first binning the mean field to a coarser grid defined by the parameter $\mathrm{ND}$, i.e., a $\mathrm{ND}^{\circ} \mathrm{xND}{ }^{\circ}$ grid, meaning that for each large bin the algorithm calculates one average value of mean SST. This coarsened mean field is then regridded back by linear interpolation onto the original grid, 

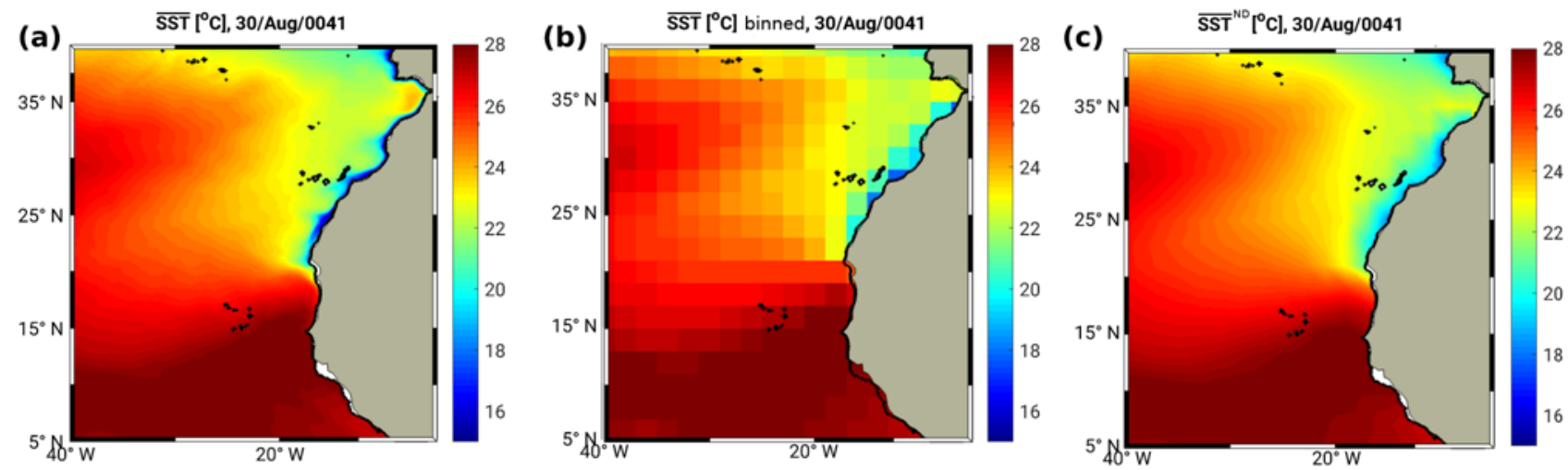

Figure S1: Example of the result of the smoothing of the mean SST field for one particular step of the reference mean field. (a) Reference mean $\overline{S S T}(x, t)$ field for the 2-day step 30-31/Aug of the 40th year of run. (b) Binned mean field for a $2 \times 2$ degree coarse grid. (c) Smoothed $\overline{S S T}^{N D}(x, t)$ field obtained after regridding of the coarse binned field onto the original grid.

obtaining a smooth mean SST field, namely $\overline{\mathrm{SST}}^{\mathrm{ND}}(\mathrm{x}, \mathrm{t})$. In step 3, the algorithm calculates the time varying SST anomalies $\Delta \mathrm{SST}(\mathrm{x}, \mathrm{t})$ as the difference between $\mathrm{SST}(\mathrm{x}, \mathrm{t})$ and $\overline{\mathrm{SST}}^{\mathrm{ND}}(\mathrm{x}, \mathrm{t})$ for each corresponding time step:

$$
\Delta \operatorname{SST}(\mathrm{x}, \mathrm{t})=\operatorname{SST}(\mathrm{x}, \mathrm{t})-\overline{\mathrm{SST}}^{\mathrm{ND}}(\mathrm{x}, \mathrm{t})
$$

In the forth step, the grid points $\mathrm{x}_{i, j}$ for which the anomalies lay below the defined threshold $\Delta \mathrm{SST}^{\mathrm{TH}}$ are marked as ones in the mask $\mathrm{M}(\mathrm{x}, \mathrm{t})$, meaning that they represent potential filaments, while the remaining points are marked as zeros:

$$
\mathrm{M}(\mathrm{x}, \mathrm{t})=\left\{\begin{array}{cc}
1 & \Delta \mathrm{SST}(\mathrm{x}, \mathrm{t}) \leq \Delta \mathrm{SST}^{\mathrm{TH}} \\
0 & \Delta \mathrm{SST}(\mathrm{x}, \mathrm{t})>\Delta \mathrm{SST}^{\mathrm{TH}}
\end{array}\right.
$$

Fifth, this "potential filament" mask is checked for overlapping eddies. If available, the results of a independent eddy-identification routine are used to set to zero any portion of the mask that overlaps with an identified eddy. If not available, the routine proceeds directly to the next step. In the final step 6, for each time step t separately, the algorithm extracts the filaments from the entire ensemble of connected domains of ones in $\mathrm{M}(\mathrm{x}, \mathrm{t})$. In order to do so, it sets to zero all the connected domains that do not touch the coast of the upwelling system of interest, in our case the north-western African coast.

The obtained mask identifies the upwelling filaments, defined as small-scale cold water structures that originate at the coast of the upwelling region. Two examples of the results of our upwelling filament identification algorithm for the Canary Upwelling System (CanUS) in different seasons are shown in Figure 2.

\section{S 1.2 Parameter sensitivity}

In the present study, we used the following set of user-defined parameters: $\mathrm{ND}=2^{\circ}, \Delta \mathrm{SST}^{\mathrm{TH}}=-0.3^{\circ} \mathrm{C}$. In the following paragraphs, we discuss the sensitivity of the algorithm to the choice of these parameters. Figure 3 provides two examples of the results obtained by halving and doubling the parameters in our setup; these alternative filament masks can be compared to the reference masks obtained for the same days using our default choice of parameters (Figure 2).

Through the smoothing of the mean reference field (ND parameter), the algorithm reduces the offshore 

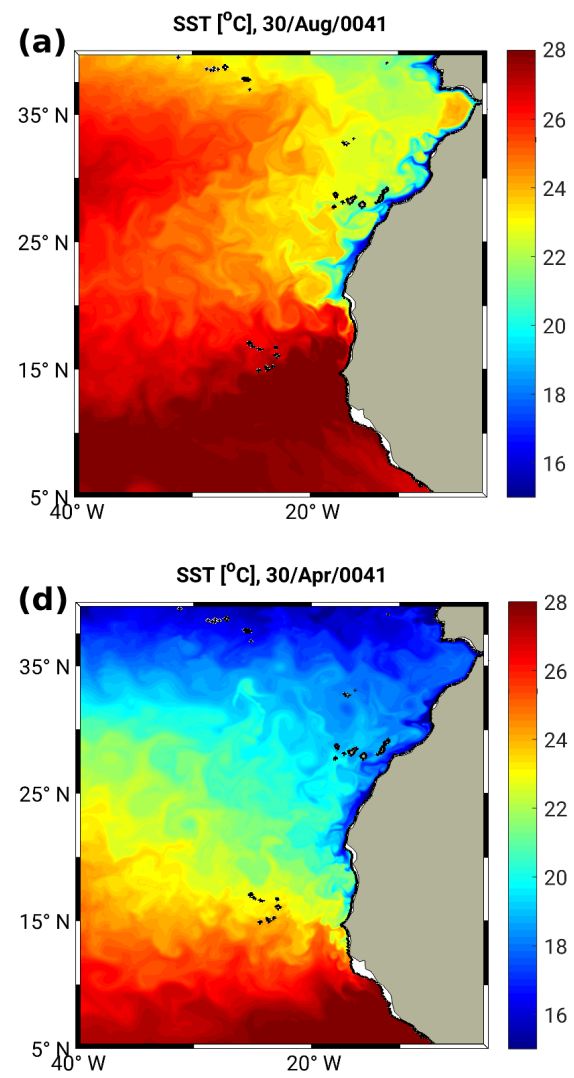
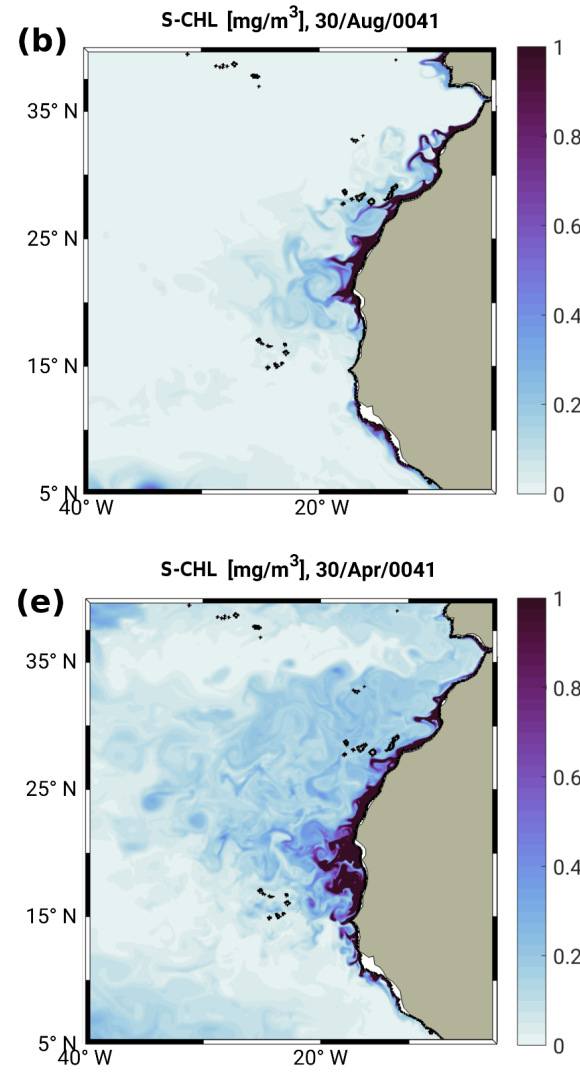

(c)

$\mathrm{ND}=2 \quad \Delta \mathrm{SST}^{\mathrm{TH}}=-0.3, \quad 30 / \mathrm{Aug} / 0041$

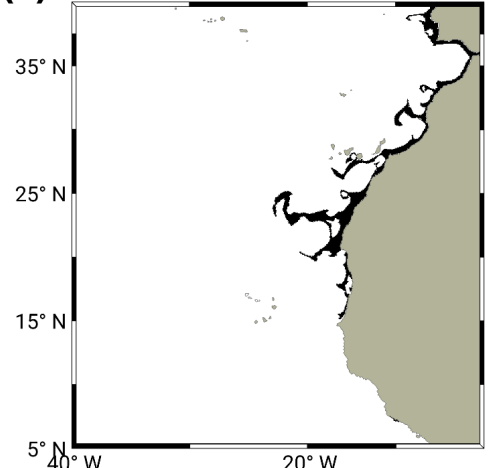

(f)

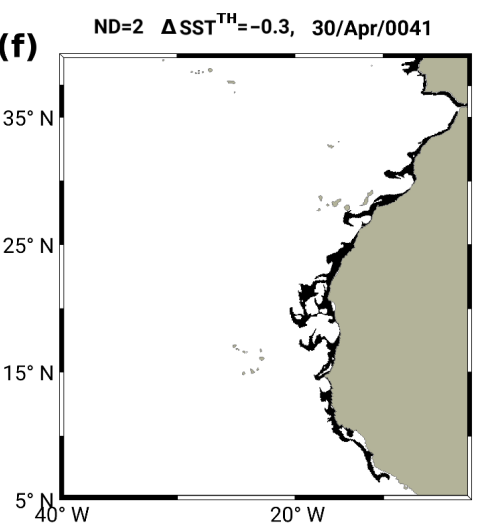

Figure S2: Examples of the resulting filament mask (excluded eddies) extracted from the SST field for upwelling conditions in the northern $(a-c)$ and southern (d-f) CanUS, respectively obtained from the 2-day SST field of the 30-31/August and the 30-31/April of the 40th year of simulation. (a) and (d): SST field; (b) and (e) S-CHL field; (c) and (f) resulting filament mask in black. Parameters: $N D=2^{\circ}, \triangle S S T^{T H}=-0.3^{\circ} \mathrm{C}$.

gradient of SST. This allows the cold-water signature of the upwelled water to clearly emerge in the $\Delta \mathrm{SST}(\mathrm{x}, \mathrm{t})$ as a pattern of negative anomalies connected to the continental coast, and therefore makes it possible to distinguish the coastally generated upwelling filaments from the offshore negative $\Delta \operatorname{SST}(\mathrm{t})$ anomalies. The use of a small value for $\mathrm{ND}$ (e.g., $\mathrm{ND}=1^{\circ}$, see Figure $3 \mathrm{a}$,e) enhances the ability of the algorithm to outline the small scale features of the filaments, but it can also result in an underestimation of the width of the filaments. For large ND (e.g., $\mathrm{ND}=4^{\circ}$, see Figure $3 \mathrm{~b}$,f), the width and, sometimes, length of the detected filaments increases substantially, while the details of the small scale structures are lost due to the stronger smoothing of the offshore gradient, which results in overall larger $\Delta \mathrm{SST}(\mathrm{t})$ values in the nearshore.

The sensitivity of the algorithm to the halving and doubling of the second user defined parameter $\Delta \mathrm{SST}^{\mathrm{TH}}$ is more substantial. The use of a loose SST threshold (e.g., $\Delta \mathrm{SST}^{\mathrm{TH}}=0.15^{\circ} \mathrm{C}$, see Figure $3 \mathrm{c}, \mathrm{g}$ ) can result in an overestimation of the length and width of the filaments, especially in regions of reduced offshore gradients such as the southern CanUS. Moreover, in the presence of widespread regions of nearly uniform SST, it can result in the detection of large-scale connected domains extending to the offshore waters. On the contrary, a strict threshold (e.g., $\Delta \mathrm{SST}^{\mathrm{TH}}=0.6^{\circ} \mathrm{C}$, see Figure $3 \mathrm{~d}, \mathrm{~h}$ ) results in the identification of extremely narrow and very short structures.

In spite of the sensitivity of the algorithm to the two parameters, the resulting masks for any of the combinations of values of ND and SST show clear similarities and identify the same set of structures in the nearshore. This confirms the overall robustness and reliability of our filament identification algorithm. 

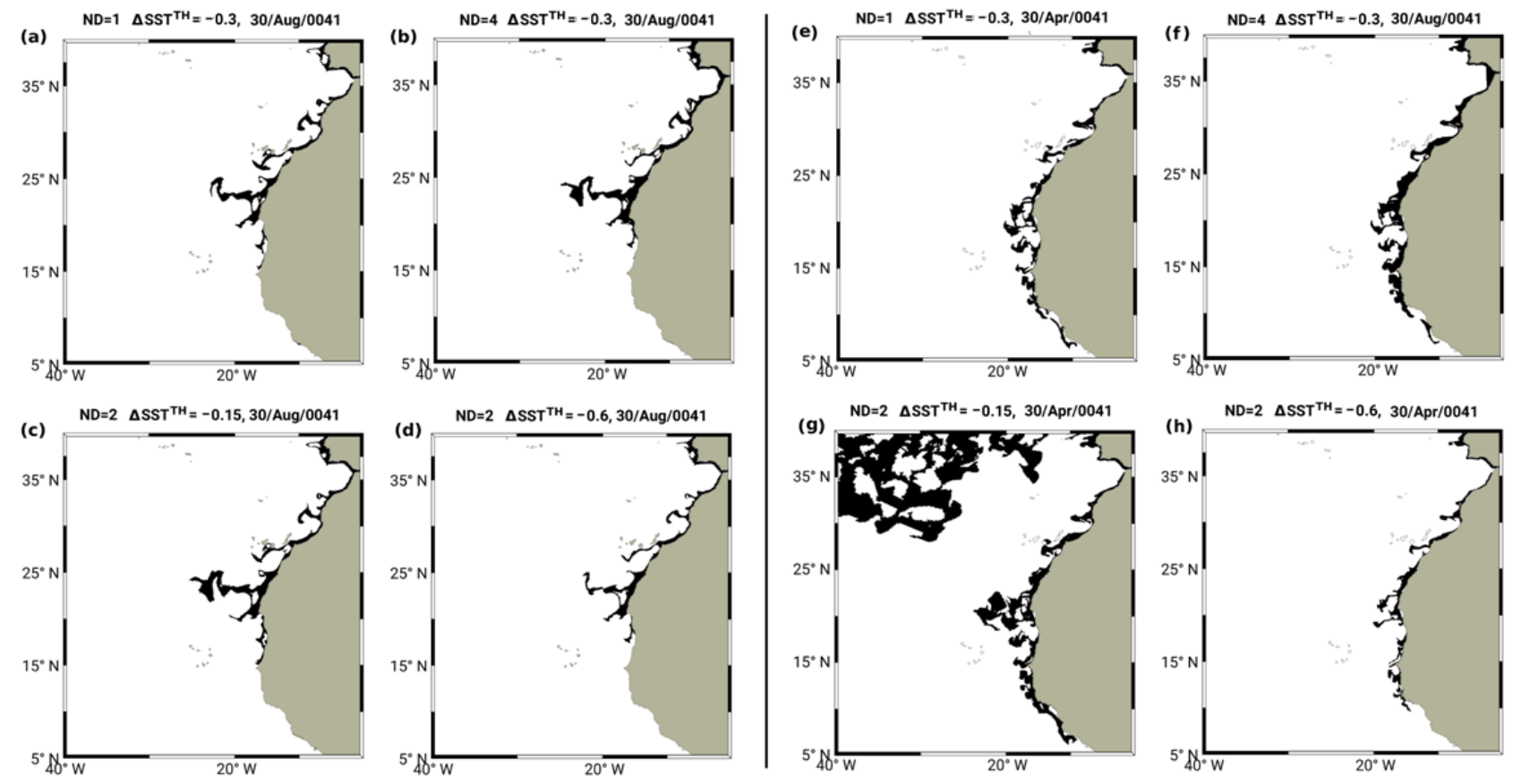

Figure S3: Examples of the resulting filament masks (excluded eddies) extracted from the SST field for upwelling conditions in the northern $(a-d)$ and southern $(e-h)$ CanUS, respectively obtained for varying values of the user-defined parameters. (a) and $(e): N D=1^{\circ}, \Delta S S T^{T H}=-0.3^{\circ} \mathrm{C} ;(\mathrm{b})$ and $(\mathrm{f}): N D=4^{\circ}, \Delta S S T^{T H}=-0.3^{\circ} \mathrm{C} ;(\mathrm{c})$ and $(\mathrm{g}): N D=2^{\circ}, \Delta S S T^{T H}=$ $-0.15^{\circ} \mathrm{C} ;(d)$ and $(h): N D=2^{\circ}, \Delta S S T^{T H}=-0.6^{\circ} \mathrm{C}$.

\section{S 1.3 Performance and consistency}

We describe here the performance of the algorithm for our choice of user-defined parameters $\mathrm{ND}=2^{\circ}, \Delta \mathrm{SST}^{\mathrm{TH}}$ $=-0.3{ }^{\circ} \mathrm{C}$. An analysis of the filament occupation in time (Figure 4) suggests that our algorithm is able to identify the known recurrent upwelling filaments that characterize the CanUS and that is able to capture differences in terms of their offshore extension (Arístegui et al., 2009).

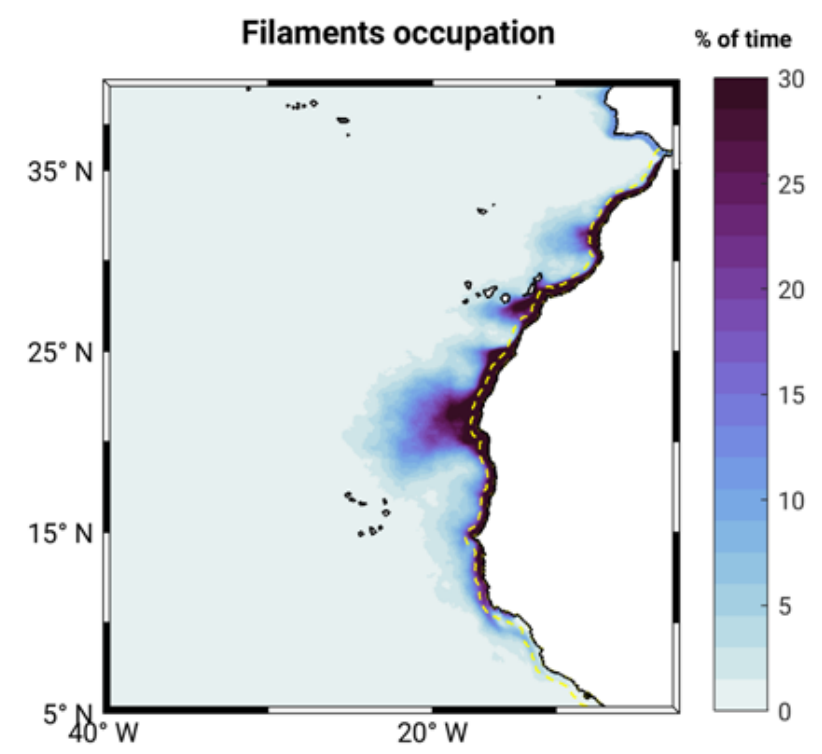

Figure S4: Percent of the total time in which each grid point is covered by a filament, according to our time varying filament mask.The yellow dashed line indicates the $50 \mathrm{~km}$ offshore boudary, strongly impacted by the cold upwelled water and often covered by the filament mask. 
Numerous comparisons between the filament mask and the SST and S-CHL fields show that our choice of the parameters provides a rather conservative estimate of the extension of the filaments, especially in the southern CanUS, with an overall high detection rate in terms of number of structures and a limited number of events of over detection. The latter are largely confined to the southern latitudes of the CanUS, especially in the proximity of the Senegalese coast and of the Cape Verde archipelago, due to the local reduced offshore gradient in SST (Figure 5). Such events are however sporadic, i.e., they never affect the filament field for long periods of time. Moreover, the portion of mask that exceeds the filament extension likely captures regions characterized by less intense flow compared to the one of the actual structures, and is therefore expected to have little impact on the fluxes.
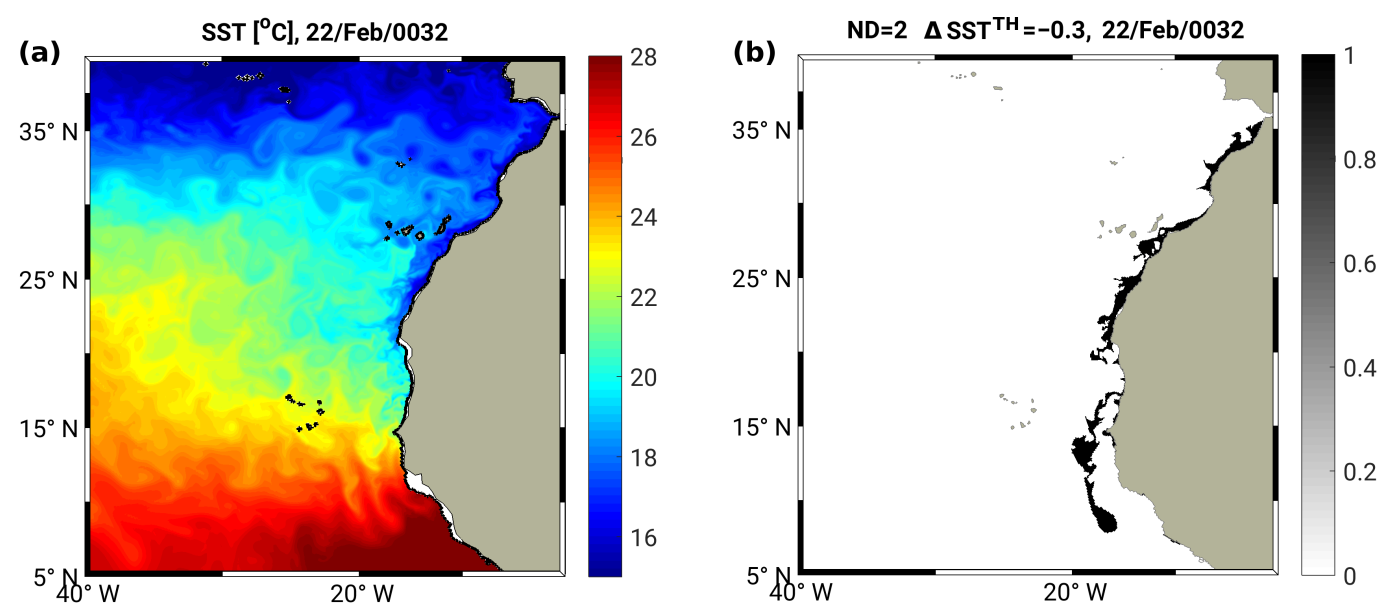

Figure S5: Example of filament overestimation.

The time evolution of the filament mask in successive time steps (Figure 6) highlights the good consistency of the algorithm performance in similar SST conditions. Identified filaments evolve smoothly in time and in space according to the natural process of elongation, meandering and retraction of the structure.

The good performance of our newly developed identification algorithm gives us confidence in our results regarding the organic carbon budget and fluxes of the filaments. The algorithm is available for future use and additional testing on different upwelling regions. Please, contact the corresponding author (Elisa Lovecchio, elisa.lovecchio@usys.ethz.ch) in this matter. 

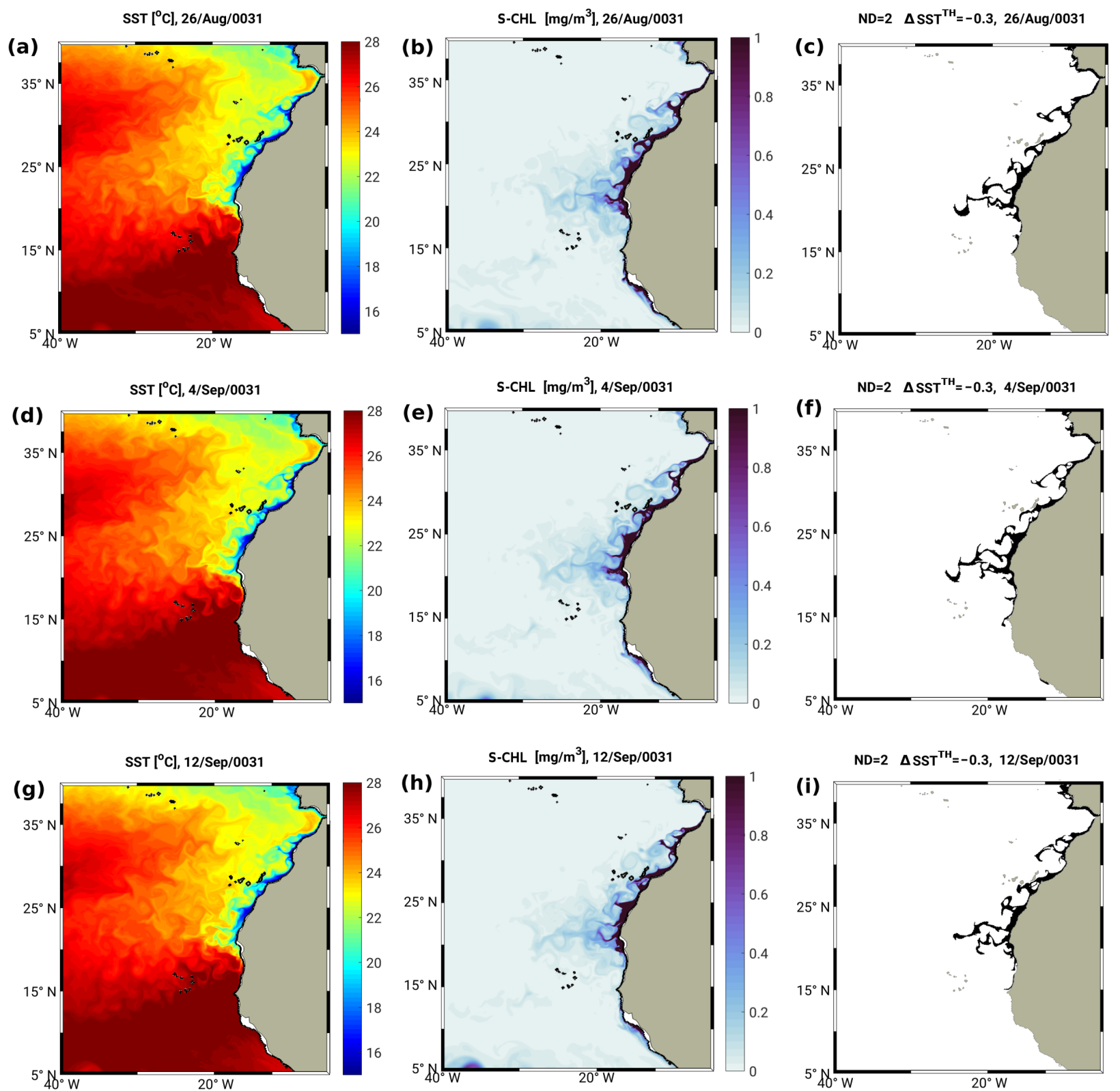

Figure S6: Evolution of the filament mask (third column) in successive time steps, namely the 2-day means referring to: (a-c) 26-27/August, (d-f) 4-5/September and ( $g-i)$ 12-13/September of the 31 st year of run. 


\section{References}

Arístegui, J., Barton, E. D., Álvarez-Salgado, X. A., Santos, M. P., Figueiras, F. G., Kifani, S., HernándezLeón, S., Mason, E., Machú, E., and Demarq, H.: Sub-regional ecosystem variability in the Canary Current upwelling, Progress in Oceanography, 83, 33-48, doi:http://dx.doi.org/10.1016/j.pocean.2009.07.031, 2009.

Cravo, A., Relvas, P., Cardeira, S., Rita, F., Madureira, M., and Snchez, R.: An upwelling filament off southwest Iberia: Effect on the chlorophyll a and nutrient export, Continental Shelf Research, 30, 1601 - 1613, doi:http://dx.doi.org/10.1016/j.csr.2010.06.007, URL http://www.sciencedirect.com/science/article/pii/s0278434310002001, 2010.

Gabric, A. J., Garcia, L., Camp, L. V., Nykjaer, L., Eifler, W., and Schrimpf, W.: Offshore export of shelf production in the Cape Blanc (Mauritania) giant filament as derived from coastal zone color scanner imagery, Journal of Geophysical Research, 98, 4697-4712, doi:10.1029/92JC01714, 1993. 